\title{
New Zealand's science-led response to the SARS-CoV-2 pandemic
}

New Zealand has avoided the major health impacts of the SARS-CoV-2 pandemic due to a strict country-wide lockdown, the end-goal of which was elimination rather than mitigation and suppression. The New Zealand government's use of scientific expertise, spanning public health, infectious diseases, genomics, modeling and immunology, has been one of the keys to the success of its SARS-CoV-2 elimination and control strategy.

\section{Jemma L. Geoghegan, Nicole J. Moreland, Graham Le Gros and James E. Ussher}

$\mathrm{T}$ he SARS-CoV-2 pandemic has posed significant public health and economic challenges to New Zealand and its Pacific neighbors. These have been met with decisive government leadership that is both focused on protecting the population and strongly informed by epidemiology, infectious disease and immunology expertise from across the science sector. Specifically, there were major concerns around the capacity of New Zealand's health system to cope in the event of widespread community transmission of SARS-CoV-2, especially in vulnerable communities. Furthermore, the lack of pharmaceutical research and development capability meant that New Zealand was totally reliant on the competitive international marketplace for sourcing and evaluating appropriate vaccines against SARS-CoV-2. The government responded by seeking to eliminate SARS-CoV-2 from the population by using highly successful public health measures and establishing a Vaccine Taskforce responsible for ensuring access to safe and effective vaccines as an exit strategy.

SARS-CoV-2 was eliminated from the community by the government instituting a strict border control regime and a stay-at-home order for all, with the exception of essential workers, in March 2020 (refs. ${ }^{1,2}$ ) (Fig. 1). The order remained in place for 49 days, with the basic reproductive number $\left(R_{0}\right)$ reduced to $<1$ during this time ${ }^{3,4}$. Through maintaining strict border restrictions and quarantine requirements, New Zealand has remained largely COVID-19 free, barring occasional rapidly controlled incursions related to the infection of border workers.

A key feature of the success in eliminating SARS-CoV-2 was the initiative shown by regional diagnostic laboratories and academic scientists, who moved early to rapidly develop and implement in-house laboratory RT-PCR (PCR with reverse transcription) tests for SARS-CoV-2. Testing

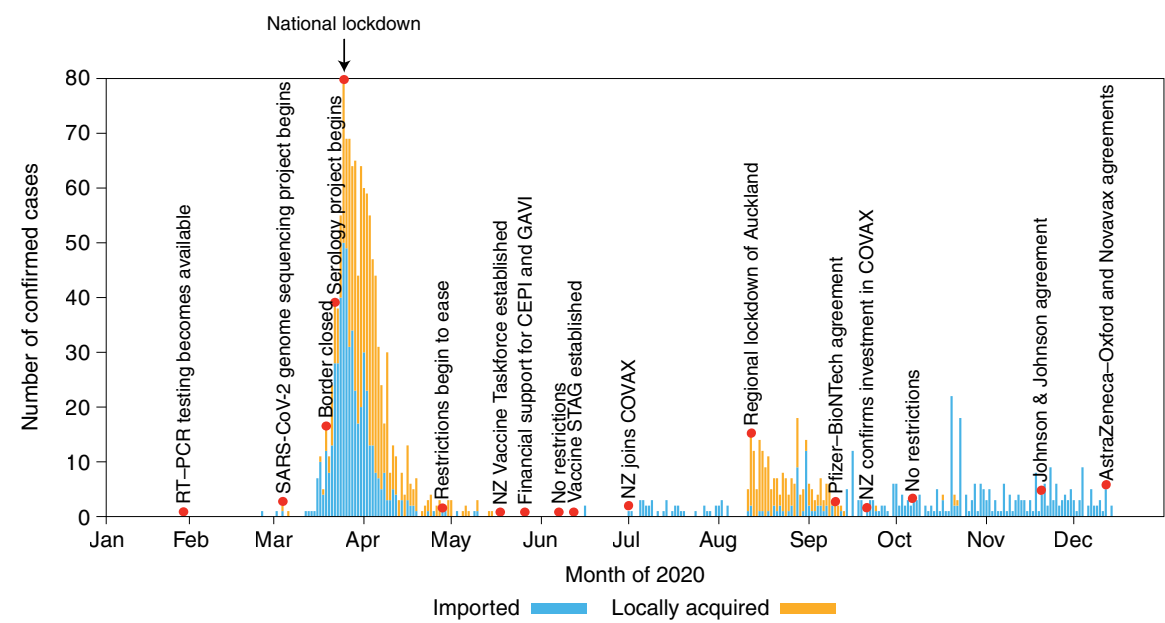

Fig. 1 | Timeline of the New Zealand government's pandemic control measures and their impact on SARS-CoV-2 cases and community transmission. The numbers of laboratory-confirmed COVID-19 cases in New Zealand that were linked to overseas travel (blue) or locally acquired (yellow) over time. Key events in New Zealand are labeled on the timeline.

became available from 31 January 2020 (Fig. 1). Laboratories then rapidly scaled up their capacity from 0.015 tests per thousand people per day in the second week of March to 1.15 tests per thousand people per day by the second week of May, just before the stay-at-home order was lifted. Testing peaked in mid-August at 4.11 tests per thousand people per day, during a limited community outbreak in Auckland. In the second week of March, four laboratories, both state funded and private, had laboratory-developed tests for SARS-CoV-2. By mid-May, twelve laboratories were offering laboratory-developed and/or commercial assays, and pooling had been introduced to manage critical reagent shortages and high testing volumes. The laboratory response was facilitated by an accreditation agency that is permissive of in-housedeveloped tests and that works with diagnostic laboratories.
Viral genomics was also critical to New Zealand's successful public health response, with genomic sequencing being used as a key tool for understanding and limiting the spread of COVID-19. By integrating genomics with epidemiological and modeling data, local transmission chains and regional spread were able to be tracked and audited in real time. When combined with geographic information, the pathways of viral spread, including from the global population, domestically and at the community level, were more easily revealed ${ }^{4,5}$. These results were used by government agencies to direct public health interventions, highlight transmission hotspots to target community testing, identify superspreading events and assess the impact of interventions such as travel restrictions and border closures. More specifically, the rapidity with which SARS-CoV-2 genomes were sequenced and analyzed aided the investigation of 
the source of infections when physical links were unclear and clarified cluster membership during community outbreaks ${ }^{6}$. This information provided to public health helped to prevent a second regional lockdown of New Zealand's largest city, avoiding significant economic costs.

Antibody testing and serological assays is another area in which New Zealand successfully leveraged strong collaborative links between research scientists and clinicians. Some of the first serological assays established were based on locally produced SARS-CoV-2 spike antigens in academic laboratories as a means to protect against potential supply chain disruption during the country-wide lockdown? ${ }^{7}$. With the rapid release of serological tests by diagnostic companies in the months that followed, validation of accuracy became a priority for academic-clinical collaborations $^{8,9}$. While serological testing is now embedded in clinical laboratories, large-scale serosurveys have not been prioritized, given the country's overall low disease prevalence. Indeed, a study of 1,127 high-risk individuals from the Southern region of New Zealand did not identify any missed infections via serology, endorsing the successful public health response during the initial lockdown ${ }^{8}$. This COVID-19-free status now provides the country with a unique opportunity to study antibody persistence from both natural infections and vaccines in the absence of immune boosting from SARS-CoV-2 re-exposure ${ }^{10}$.

The initial uncertainty of access to leading SARS-CoV-2 vaccine candidates under development internationally was a significant challenge for the small economies of New Zealand and the Pacific countries. From the outset, the approach of the national Vaccine Taskforce was broad, including investing in the international COVAX facility; pursuing bilateral advance purchase agreements with major vaccine developers; and establishing a national preclinical and clinical vaccine evaluation platform, Vaccine Alliance Aotearoa New Zealand - Ohu Kaupare Huaketo (VAANZ). The cross-ministerial Vaccine Taskforce is supported by a Science and Technical Advisory Group (STAG) that includes representatives from all areas of the clinical, science and health sphere. The STAG ensures that the vaccine strategy is not only informed by the latest data and a range of scientific perspectives, but, with a New Zealand-specific lens applied, that the vaccine portfolio and roll-out are appropriate for the New Zealand setting, with a particular focus on ensuring equitable access for indigenous Māori and Pacific populations.

At the time of writing, there is no community transmission, the economy has bounced back from the forecast $23.5 \%$ drop in GDP to be up $0.4 \%$ on the previous year's December performance ${ }^{11}$, and New Zealand is on track to vaccinate its population, the Realm of New Zealand (Tokelau, Niue and Cook Islands) and its neighbors (Samoa, Tonga and Tuvalu) with at least four of the current leading SARS-CoV-2 vaccines, should their governments and communities wish to take these up.
Jemma L. Geoghegan 1,2,3,

Nicole J. Moreland ${ }^{3,4}$, Graham Le Gros (D) 5,6凶 and James E. Ussher (ID) 1,3,6,7

${ }^{1}$ Department of Microbiology and Immunology, University of Otago, Dunedin, New Zealand. ${ }^{2}$ Institute of Environmental Science and Research, Wellington, New Zealand. ${ }^{3}$ Maurice Wilkins Centre for Molecular Biodiscovery, University of Auckland, Auckland, New Zealand. ${ }^{4}$ Department of Molecular Medicine and Pathology, University of Auckland, Auckland, New Zealand. ${ }^{5}$ Malaghan Institute of Medical Research, Wellington, New Zealand.

${ }^{6}$ Ohu Kaupare Huaketo, Vaccine Alliance Aoteoroa New Zealand, Wellington, New Zealand. ${ }^{7}$ Southern Community Laboratories, Dunedin, New Zealand.

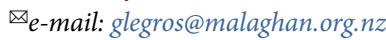

Published online: 24 February 2021

https://doi.org/10.1038/s41590-021-00872-X

References

1. Baker, M. G., Wilson, N. \& Anglemyer, A. N. Engl. J. Med. 383, e56 (2020).

2. Jefferies, S. et al. Lancet Public Health https://doi.org/10.1016/S24682667(20)30225-5 (2020).

3. Binny, R. N. et al. Preprint at medRxiv https://doi.org/10.1101/2020 08.10.20172320 (2020)

4. Geoghegan, J. L. et al. Nat. Commun. 11, 6351 (2020).

5. Swadi, T. et al. Emerg. Infect. Dis. https://doi.org/10.3201/eid2703. 204714 (2021).

6. Geoghegan, J. L. et al. Preprint at medRxiv https://doi.org/10.1101/ 2020.10.28.20221853 (2020).

7. McGregor, R. et al. PeerJ 8, e9863 (2020).

8. Craigie, A. et al. Preprint at medRxiv https://doi.org/10.1101/2020. 10.20.20215616 (2020).

9. Fox-Lewis, S. et al. NZ Med. J. 133, 127-131 (2020).

10. Whitcombe, A. L. et al. Preprint at medRxiv https://doi.org/10.1101/ 2020.12.10.20246751 (2020)

11. The Treasury. Weekly Economic Update - 18 December 2020 (2020); https://www.treasury govt.nz/publications/weu/ weekly-economic-update-18-december-2020-html

Competing interests

The authors declare no competing interests. 